\title{
THE GENERALIZED AHLFORS-HEINS THEOREM IN CERTAIN $d$-DIMENSIONAL CONES
}

\author{
MATTS ESSÉN and JOHN L. LEWIS
}

\section{Notation.}

Let $\Omega$ be an open set in $\mathrm{R}^{d}, d \geqq 2$, and let $\partial \Omega$ be its boundary. The closure of a set $D$ is denoted by $\bar{D}$. In cartesian coordinates a point $x$ is denoted by $\left(x_{1}, \ldots, x_{d}\right)$. Let $|x|$ be the Euclidean norm of $x$. Also let $e(x)$ be the radial projection of $x$ onto the unit sphere. If the function $u$ is defined in $\Omega$ and $y \in \partial \Omega$, we define

$$
u(y)=\lim \sup u(x), \quad x \rightarrow y, x \in \Omega .
$$

We also introduce

$$
M(r)=M(r, u)=\sup u(x), \quad|x|=r, x \in \Omega,
$$

and $M(r)^{+}=\max \{M(r), 0\}$.

A system of spherical coordinates for $x$ is given by

$$
r=|x|, \quad x_{1}=r \cos \theta_{1}, \quad x_{d}=r \prod_{j=1}^{d-1} \sin \theta_{j},
$$

and if $d>2$,

$$
x_{i}=r \cos \theta_{i} \prod_{j=1}^{i-1} \sin \theta_{j}, \quad 2 \leqq i \leqq d-1 .
$$

Here, $0 \leqq \theta_{d-1} \leqq 2 \pi$, and if $d>2$, then $0 \leqq \theta_{i} \leqq \pi, 1 \leqq i \leqq d-2$. Relative to this system, the Laplace operator $\Delta$ may be written

$$
\Delta=r^{1-d} \frac{\partial}{\partial r}\left(r^{d-1} \frac{\partial}{\partial r}\right)+r^{-2} \delta
$$

where the Beltrami operator $\delta$ is given by

$$
\delta=\sum_{k=1}^{d-1} T^{-1} \frac{\partial}{\partial \theta_{k}}\left(T T_{k}^{-1} \frac{\partial}{\partial \theta_{k}}\right) .
$$

Here $T=\Pi_{j=1}^{d-1}\left(\sin \theta_{j}\right)^{d-1-j}, T_{1}=1$, and if $d>2, T_{k}=\Pi_{j=1}^{k-1}\left(\sin \theta_{j}\right)^{2}, 2 \leqq$ $k \leqq d-1$. 
If $\alpha$ is given, $0<\alpha<\pi$, and $d \geqq 3$, let $K(\alpha)$ be the cone $\left\{x: 0 \leqq \theta_{1}<\alpha\right\}$. If $d=2$ let $K(\alpha)$ be the sector $\left\{x:\left|\theta_{1}\right|<\alpha\right\}$. We also introduce the polar cap

$$
S(\alpha)=K(\alpha) \cap\{x:|x|=1\} .
$$

If $f$ is a function defined in $K(\alpha)$, we shall write $f(r, 0)$ for the value of $f$ at the point whose spherical coordinates are $r$ and $\theta_{1}=0$. Moreover, if $f(x)=f(y)$ whenever $x$ and $y$ have the same $r, \theta_{1}$, coordinates we shall write $f\left(r, \theta_{1}\right)$ for the value of $f$ at any point $x$ whose first two spherical coordinates are $r$ and $\theta_{1}$.

Consider in $S(\alpha)$ the boundary value problem

$$
\delta g+\mu g=0, \quad g=0 \text { on } \partial S(\alpha),
$$

where we assume that $g$ is continuous in $\overline{S(\alpha)}$ and that $\delta g$ is continuous in $S(\alpha)$. The classical theory of the problem (1.2) goes back to Bouligand (see J. Lelong-Ferrand [10] for further references). Let $\mu_{1}>0$ be the first eigenvalue and $\psi_{1}$ the unique corresponding eigenfunction satisfying

$$
\psi_{1}(1,0)=1 .
$$

Next let $\varrho$ be the positive root of the equation

$$
\varrho(\varrho+d-2)=\mu_{1} \text {. }
$$

We also consider for fixed $\lambda, 0<\lambda<1$, and for the same class of functions as in (1.2) the boundary value problem

$$
\delta g+\varrho \lambda(\varrho \lambda+d-2) g=0, \quad g=1 \text { on } \partial S(\alpha) .
$$

This problem has a unique solution which we denote by $\psi_{\lambda}$. We note that $\psi_{\lambda}(x)=\psi_{\lambda}(y)$ whenever $x, y \in S(\alpha)$ have the same $r, \theta_{1}$, coordinates, as follows from the symmetry of $S(\alpha)$. Using this fact and the minimum principle (see $[3$, p. 326]) we find that

$$
1<\psi_{\lambda}(x)<\psi_{\lambda}(1,0)=C(\lambda)^{-1}, \quad x \in S(\alpha), x \neq(1,0, \ldots, 0) .
$$

With $C(\lambda)$ as in (1.5), we define

$$
H_{\lambda}(x)=C(\lambda) \psi_{\lambda}(e(x))|x|^{e^{\lambda}}, \quad x \in K(\alpha) .
$$

It follows from (1.1) that $H_{\lambda}$ is harmonic in $K(\alpha)$. Moreover, from (1.5) we see that

$$
\begin{gathered}
H_{\lambda}(1,0)=1, \\
H_{\lambda}(y)=C(\lambda) H_{\lambda}(|y|, 0)=C(\lambda) M\left(|y|, H_{\lambda}\right), \quad y \in \partial K(\alpha) .
\end{gathered}
$$




\section{The main result.}

Let $u$ be subharmonic in $K(\alpha)$ and suppose for given $\lambda, 0<\lambda<1$, that $u$ satisfies either

$$
u(y) \leqq C(\lambda) u(|y|, 0), \quad y \in \partial K(\alpha), u(0) \leqq 0,
$$

or the following conditions:

$$
\begin{gathered}
u(y) \leqq C(\lambda) M(|y|), \quad y \in \partial K(\alpha)-\{0\}, \\
u(y)<\infty, \quad y \in \partial K(\alpha) .
\end{gathered}
$$

Here $C(\lambda)$ is as in (1.5). Then we shall prove

Theorem 1. Let $\lambda$ be given, $0<\lambda<1$. Let $u \neq-\infty$ be subharmonic in $K(\alpha)$ and satisfy (2.1). If

$$
\liminf _{r \rightarrow \infty} r^{-\lambda \varrho} M(r)<\infty,
$$

then for some $\beta,-\infty<\beta<\infty$, we have

(A). Except when $e(x) \in S(\alpha)$ belongs to a set of capacity zero,

$$
\lim _{r \rightarrow \infty} r^{-\lambda \ell} u(r e(x))=\beta \psi_{\lambda}(e(x)) .
$$

(B). There is an exceptional set $F_{0}$ of spheres whose radii $r_{i}$ and distances $R_{i}$ from the origin satisfy $\sum_{i=1}^{\infty}\left(r_{i} / R_{i}\right)^{d-1}<\infty$, for which

$$
\lim _{x \rightarrow \infty}\left[|x|^{-\lambda \varrho} u(x)-\beta \psi_{\lambda}(e(x))\right]=0
$$

uniformly in $K(\alpha)-F_{0}$.

Theorem 2. Let $u$ be subharmonic in $K(\alpha)$ and satisfy (2.2) and (2.3) for fixed $\lambda, 0<\lambda<1$. If

$$
u\left(x_{0}\right)>0 \text { for some } x_{0} \in K(\alpha),
$$

and if $\liminf _{r \rightarrow \infty} r^{-\lambda o} M(r)<\infty$, then (A) and (B) of Theorem 1 hold for some $\beta$ satisfying $0<\beta<\infty$.

Theorems 1 and 2 have an important limiting case as $\lambda \rightarrow 1$. Indeed, if we put $C(1)=\lim _{\lambda \rightarrow 1} C(\lambda)=0$, and if $\psi_{1}$ is as in (1.3), then our result is also true for $\lambda=1$. In order to describe earlier work, let us say that an author has considered the case $\alpha_{1}<\alpha$ if he has proved that the limit in (2.4b) exists, apart from an exceptional set, uniformly in each cone $K\left(\alpha_{1}\right), 0<\alpha_{1}<\alpha$.

The case $\lambda=1, \alpha_{1}<\alpha$, was considered in $\mathrm{R}^{2}$ by Ahlfors and Heins [1]. The extension to the case $\lambda=1, \alpha_{1}=\alpha$, was given by Hayman [12]. In 
higher dimensions the case $\lambda=1, \alpha_{1}<\alpha$, was first treated by J. LelongFerrand ([9], [10], [11]). The extension to the case $\lambda=1, \alpha_{1}=\alpha$, is due to Azarin [2].

In the sequel, we shall suppose that $d \geqq 3$ since this simplifies our notation. Actually in $\mathrm{R}^{2}$, Theorems 1 and 2 hold for $0<\lambda<2$ if $C(\lambda)=$ $\cos \left(\frac{1}{2} \pi \lambda\right)$. The case $0<\lambda<2, \alpha_{1}<\alpha$, may be found in Essén [6] and Lewis [17]. The extension to the case $0<\lambda<2, \alpha_{1} \leqq \alpha$, may be obtained by essentially the same arguments as those used when $d \geqq 3$.

A preliminary version of Theorem 1 in $R^{3}$ is given in Essén [8]. An outline of the proof of Theorem 2 in the case $d=3$ is in [7]. We have only been able to prove that Theorem 2 is true in a circular cone. We would like to mention that Theorem 1 is valid in more general cones. The proof is, apart from a few steps, similar to the proof of Theorem 2 which is given in the present paper. We also note that Dahlberg [4] has considered subharmonic functions which satisfy the hypotheses of Theorem 2 in more general cones. For the cones $K(\alpha)$, his conclusion is that $r^{-\lambda e} M(r)$ tends to a positive limit as $r \rightarrow \infty$. This conclusion also follows from our result, as is easily seen.

In general we do not have an explicit formula for the constant $C(\lambda)$. However, if $d=2$, then as mentioned above $C(\lambda)=\cos \frac{1}{2} \pi \lambda$. If $d>2$ and $\alpha=\frac{1}{2} \pi$, we can use the Poisson integral formula for $K\left(\frac{1}{2} \pi\right)$ to conclude that

$$
C(\lambda)^{-1}=2 \sigma_{d-1} \sigma_{d}^{-1} \int_{0}^{\infty} t^{d-2+\lambda}\left(1+t^{2}\right)^{-d / 2} d t,
$$

where $\sigma_{d}$ is the surface area of the $d$ dimensional unit sphere. In terms of the $\Gamma$ function,

$$
\begin{gathered}
\sigma_{d}=2 \pi^{d / 2} / \Gamma(d / 2), \\
C(\lambda)=\pi^{\frac{1}{2}} \Gamma\left(\frac{1}{2}(d-1)\right) \Gamma\left(\frac{1}{2}(d-1+\lambda)\right)^{-1} \Gamma\left(\frac{1}{2}(1-\lambda)\right)^{-1} .
\end{gathered}
$$

In the proofs, methods developed by the authors in $\mathrm{R}^{2}$ are used (see [6], [17]). There is one important new feature: the use of the technique of Azarin [2] and Hayman [12], which gives a more precise description of the exceptional set.

\section{A harmonic majorant.}

We first note that Theorem 1 is a consequence of Theorem 2. Indeed, let $u$ be as in Theorem 1. If $B$ is large enough, the function $u_{1}=u+B H_{\lambda}$ satisfies (2.2), (2.3), and (2.5). Since $u(0) \leqq 0$, we see that $u_{1}$ satisfies the hypotheses of Theorem 2. It follows that (A) and (B) of Theorem 1 are true for $u_{1}$ and there upon for $u$. Hence it suffices to prove Theorem 2. We shall want the following lemma. 
LEMMA 1. Let $h$ be subharmonic and bounded above in $K(\alpha) \cap\{x:|x|<R\}$, $0<R<\infty$. If

$$
h(y) \leqq C(\lambda) M(|y|, h)^{+}, \quad 0<|y|<R, y \in \partial K(\alpha),
$$

then $M(r, h)^{+}$is a nondecreasing function which is also a convex function of $r^{2-d}$ on $(0, R)$. In particular, if $d M(r, h)^{+} / d r$ is the left hand derivative of $M(r, h)^{+}$, then $r^{d-1} d M(r, h)^{+} / d r$ is a nondecreasing function on $(0, R)$.

Proof. For a corresponding lemma in $R^{2}$, see Lewis [16, Lemma 1]. For $d>2$ the proof is essentially the same. It follows from the convexity that if $0<t<s$, then $\left(M(s)^{+}-M(t)^{+}\right) /\left(t^{2-d}-s^{2-d}\right)$ is increasing in each variable separately and locally bounded. Thus the last part of the lemma follows by applying Heins [13, p. 79, ex. 1].

We now claim that

$$
0<\sup _{r>0} r^{-\lambda e} M(r) \leqq C(\lambda)^{-1} \liminf _{r \rightarrow \infty} r^{-\lambda e} M(r)<\infty,
$$

where $M(r)=M(r, u)$. To prove (3.1) consider

$$
h(x)=u(x)-R^{-\lambda_{e}} C(\lambda)^{-1} M(R)^{+} H_{\lambda}(x)
$$

when $x \in K(\alpha) \cap\{x:|x|<R\}$. It follows from (2.2), (2.3), and (1.7) that $h$ satisfies the hypotheses of Lemma 1 . Hence $M(r, h)^{+}$is nondecreasing. Since

$$
h(x) \leqq 0, \quad x \in K(\alpha) \cap\{x:|x|=R\},
$$

$h$ is nonpositive and hence

$$
r^{-\lambda e} M(r) \leqq C(\lambda)^{-1} R^{-\lambda e} M(R)^{+}
$$

when $0<r<R$. Since (2.5) holds, $(3.1)$ is proved.

Next we shall study a particular harmonic majorant of $u$. For this purpose, let $g(\cdot, y)$ denote Green's function for $K(\alpha)$ with pole at $y$. Also let $\partial g(x, y) / \partial n$ denote the inner normal derivative of $g(x, \cdot)$ evaluated at the point $y \in \partial K(\alpha)$. We note for $a>0$ that

$$
\begin{aligned}
& g(a x, a y)=a^{2-d} g(x, y), \quad x, y \in K(\alpha), \\
& \partial g(a x, a y) / \partial n=a^{1-d} \partial g(x, y) / \partial n, \quad y \in \partial K(\alpha), x \in K(\alpha), \\
& |y|^{d} \partial g(x, y) / \partial n=\partial g\left(x, y /|y|^{2}\right) / \partial n, \quad y \in \partial K(\alpha)-\{0\}, x \in S(\alpha) \text {. }
\end{aligned}
$$

In (3.4) we have used the Kelvin transformation (see Helms [14, p. 36]).

Let $\sigma$ denote Lebesgue measure on $\partial K(\alpha)$. Let the measure $\mu$ be defined on $\partial K(\alpha)$ by

$$
t^{d-2} d t d \mu(y)=d \sigma(y), \quad|y|=t .
$$


From the symmetry of $K(\alpha)$ it follows for fixed $x \in K(\alpha)$ that

$$
B\left(t, \theta_{1}\right)=\int_{\partial K(\alpha) \cap\{|y|=t\}} \partial g(e(x), y) / \partial n d \mu(y)
$$

depends only on $t$ and the $\theta_{1}$ coordinate of $x$. If $|x|=r$, it follows from (3.3) that

$$
\int_{\partial K(\alpha) \cap\{|y|=t\}} \partial g(x, y) / \partial n d \mu(y)=r^{1-d} B\left(t / r, \theta_{1}\right) .
$$

Moreover, using (3.4) we obtain

$$
t^{d} B\left(t, \theta_{1}\right)=B\left(1 / t, \theta_{1}\right) .
$$

We now want to prove that there is a harmonic majorant of $u$ in $K(\alpha)$ whose value at $y \in \partial K(\alpha)$ is $C(\lambda) M(|y|)^{+}$. For this purpose consider for a given positive integer $n$ the unique, bounded, harmonic function $v_{n}$ in $K(\alpha)$ whose boundary values are

$$
\begin{aligned}
& v_{n}(y)=C(\lambda) M(|y|)^{+}, \quad 0<|y|<n, y \in \partial K(\alpha), \\
& =0 \quad, \quad|y|>n, \quad y \in \partial K(\alpha) .
\end{aligned}
$$

It is well-known (see Helms [14, p. 76]) that

$$
v_{n}(x)=C(\lambda)\left((d-2) \sigma_{d}\right)^{-1} \int_{\partial K(\alpha) n\{|y|<n\}} M(|y|)+\partial g(x, y) / \partial n d \sigma(y),
$$

where $\sigma_{d}$ is given by (2.6). In terms of the notation in (3.5), and with $v_{n}(x)=v_{n}\left(r, \theta_{1}\right)$, the above integral may be written

$$
\begin{aligned}
v_{n}\left(r, \theta_{1}\right)=A(\lambda) \int_{0}^{n} M(t) & +B\left(t / r, \theta_{1}\right) r^{1-d} t^{d-2} d t \rightarrow \\
& \rightarrow A(\lambda) \int_{0}^{\infty} M(r s)^{+} B\left(s, \theta_{1}\right) s^{d-2} d s,
\end{aligned}
$$

as $n \rightarrow \infty$. Here $A(\lambda)=C(\lambda) /\left\{(d-2) \sigma_{d}\right\}$. The sequence $\left\{v_{n}\right\}_{1}^{\infty}$ is nondecreasing and so either $v=\lim _{n \rightarrow \infty} v_{n}$ is harmonic or identically $+\infty$. The latter possibility cannot occur since it follows from (3.1) that

$$
v_{n} \leqq H_{\lambda} \sup _{r>0} r^{-\lambda \varrho} M(r)<\infty .
$$

It is also clear that $v$ is a harmonic majorant of $u$ as follows from a Phragmen-Lindelöf type argument (see Heins [13, p. 111]). We omit the proof.

Finally we show that

$$
v(r, 0)=M(r, v), \quad 0<r<\infty .
$$

Using (3.7), the last part of Lemma 1, and the Monotone Convergence Theorem, we first find that

$$
r^{d-1} \partial v\left(r, \theta_{1}\right) / \partial r=A(\lambda) \int_{0}^{\infty}(r s)^{d-1}\left\{d M(r s)^{+} / d(r s)\right\} B\left(s, \theta_{1}\right) d s .
$$


By Lemma 1, the function $r^{d-1} d M(r)^{+} / d r$ is nondecreasing and so has a derivative almost everywhere on $(0, \infty)$. From this fact, the above equality, and the Fatou Lemma, we deduce

$$
\frac{\partial}{\partial r}\left[r^{d-1} \frac{\partial}{\partial r} v\left(r, \theta_{1}\right)\right] \geqq 0 \quad 0<r<\infty .
$$

Since $v$ is harmonic it follows from (1.1) that $\delta v \leqq 0$. (3.8) is now an immediate consequence of the symmetry of $v$ and the minimum principle $[3$, p. 326].

\section{A convolution inequality.}

Since $v$ is a harmonic majorant of $u$, we have from (3.7) and (3.8) that

$$
r^{-\lambda_{\ell}} M(r)^{+} \leqq r^{-\lambda_{\ell}} v(r, 0)=A(\lambda) \int_{0}^{\infty}(r s)^{-\lambda_{\ell}} M(r s)^{+} B(s, 0) s^{d+\lambda_{\ell}-2} d s .
$$

After the change of variables $r=e^{x}, s=e^{-y}$, we obtain

$$
\Phi(x) \leqq \Phi * L(x), \quad x \in \mathrm{R},
$$

where

$$
\begin{gathered}
\Phi(x)=e^{-\lambda_{\ell} x} M\left(e^{x}\right)^{+}, \\
L(x)=A(\lambda) \exp \{(1-d-\lambda \varrho) x\} B\left(e^{-x}, 0\right), \\
\int_{-\infty}^{\infty} L(x) d x=H_{\lambda}(1,0)=1
\end{gathered}
$$

(see (1.6)).

The above convolution inequality has been studied by Essén ([5], [6]). In particular if $L$ satisfies

$$
\int_{-\infty}^{\infty} y L(y) d y \neq 0,
$$

then it follows from Essén [5] that

$$
\begin{gathered}
\lim _{x \rightarrow \infty} \Phi(x)=\lim _{r \rightarrow \infty} r^{-\lambda e} M(r)^{+} \text {exists }, \\
\int_{0}^{\infty}(\Phi-\Phi * L)(y) d y=\int_{1}^{\infty} t^{-1-\lambda e}\left[M(t)^{+}-v(t, 0)\right] d t>-\infty .
\end{gathered}
$$

(4.4) follows from the fact that $L(-x)>L(x), x>0$ (see (3.6)).

In the next section, we shall need the following lemma of Azarin [2, Lemma 1]. If $f$ and $h$ are nonnegative functions in $K(\alpha)$, let us say that $f \approx h$ if there exist positive constants $C_{1}$ and $C_{2}$ such that $C_{1} f \leqq h \leqq C_{2} f$.

Lemma 2. Let $\psi_{1}$ and $\varrho$ be as in (1.3) and (1.4). If $0<2|x| \leqq|y|$, we have

(a) $\psi_{1}(e(x)) \psi_{1}(e(y))(|x| /|y|)^{\rho}|y|^{2-d} \approx g(x, y), \quad x, y \in K(\alpha)$,

(b) $\psi_{1}(e(x))\left(\partial \psi_{1}(e(y)) / \partial n\right)(|x| /|y|)^{e}|y|^{1-d} \approx \partial g(x, y) / \partial n$,

$$
x \in K(\alpha), y \in \partial K(\alpha),
$$


while if $0<2|y| \leqq|x|$,

(c) $\psi_{1}(e(x)) \psi_{1}(e(y))(|y| /|x|)^{\rho}|x|^{2-d} \approx g(x, y), \quad x, y \in K(\alpha)$,

(d) $\psi_{1}(e(x))\left(\partial \psi_{1}(e(y)) / \partial n\right)(|y| /|x|)^{\varrho}|x|^{2-d}|y|^{-1} \approx \partial g(x, y) / \partial n$, $x \in K(\alpha), y \in \partial K(\alpha)$.

Proof. For completeness we indicate how the lemma can be proved for $d \geqq 6$. Consider the expansion of $g$ in terms of normalized eigenfunctions $\left\{\varphi_{n}\right\}_{1}^{\infty}$ of (1.2) which has been given by J. Lelong-Ferrand $\left[10\right.$, p. 341]. If the corresponding eigenvalues are $\left\{\mu_{n}\right\}_{1}^{\infty}$, there exists a constant $A$ only depending on $\alpha$ and $d$ such that

(i) $0<\mu_{n} \leqq A n^{(d-3) / 2}$,

(ii) $\psi_{1}(x) \approx \varphi_{1}(x) \approx \operatorname{dist}(x, \partial S(\alpha)), \quad x \in S(\alpha)$,

(iii) $\left|\varphi_{n}(x)\right| \leqq A \mu_{n}{ }^{(d+1) / 4} \operatorname{dist}(x, \partial S(\alpha))$.

Lemma 2 is an immediate consequence of (i), (ii), and (iii). If $2 \leqq d \leqq 5$ similar estimates are valid (see section 8 ).

As to proofs (i) and (ii) are well-known (see [3]). As to (iii), this inequality is valid for the normalized eigenfunctions of any uniformly elliptic operator with Hölder continuous coefficients. A proof is given in section 8. We do not know if the exponent occurring in (iii) is best possible.

\section{An estimate of the Riesz mass.}

Using (3.1) and (4.5), we see that

$$
\lim _{r \rightarrow \infty} r^{-\lambda_{l}} M(r)=\beta, \quad 0<\beta<\infty .
$$

From the definition of $v$ and a Phragmén-Lindelöf argument, it follows that

$$
\lim _{x \rightarrow \infty}\left[|x|^{-\lambda_{e}} v(x)-\beta C(\lambda) \psi_{\lambda}(e(x))\right]=0
$$

uniformly in $K(\alpha)$. Hence it suffices to prove (A) and (B) of Theorem 1 for $-p=u-v$. The function $p$ is nonnegative and superharmonic in $K(\alpha)$. Moreover from Lemma 1 and (2.5) we see that for $r$ large enough, there exists $x_{r} \in K(\alpha),\left|x_{r}\right|=r$, such that $u\left(x_{r}\right)=M(r, u)=M(r)^{+}$. Hence for such values of $r$,

$$
p\left(x_{r}\right)=v\left(x_{r}\right)-u\left(x_{r}\right) \leqq v(r, 0)-M(r)^{+} .
$$

From (1.5) and (5.1)-(5.3), it is clear that

$$
\lim _{r \rightarrow \infty} r^{-\lambda e} p\left(x_{r}\right)=0,
$$


and that for any $\delta, 0<\delta<\alpha$, there exists $T$ for which

$$
x_{r} \in K(\delta), \quad r>T .
$$

Using (4.6), (5.3), and (5.5), we deduce that if $\delta$ is given, $0<\delta<\alpha$, then

$$
\int_{T}^{\infty} w(r) r^{-1-\lambda e} d r<\infty,
$$

where $w(r)=\inf p(x),|x|=r, x \in K(\delta)$. Using (5.4) and (5.5) we obtain as in Azarin [2, Theorem 1], a Riesz representation formula for $p$ :

$$
p(x)=\int_{\partial K(\alpha)} \partial g(x, y) / \partial n d \gamma(y)+\int_{K(\alpha)} g(x, y) d \xi(y), \quad x \in K(\alpha),
$$

where $\gamma$ and $\xi$ are positive Borel measures on $\partial K(\alpha)$ and $K(\alpha)$, respectively.

A consequence of (5.6) and Lemma 2 is that

$$
\begin{aligned}
& \int_{K_{1}} \psi_{1}(e(y))|y|^{e} d \xi(y)+\int_{K_{2}} \psi_{1}(e(y))|y|^{2-d-\lambda e} d \xi(y)<\infty, \\
& \int_{\Sigma_{1}} \partial \psi_{1}(e(y)) / \partial n|y|^{e-1} d \gamma(y)+\int_{\Sigma_{2}} \partial \psi_{1}(e(y)) / \partial n|y|^{1-d-\lambda e} d \gamma(y)<\infty
\end{aligned}
$$

where $K_{1}=K(\alpha) \cap\{|y|<2 T\}, K_{2}=K(\alpha) \cap\{|y| \geqq 2 T\}, \quad \Sigma_{1}=\partial K_{1} \cap \partial K(\alpha)$ and $\Sigma_{2}=\partial K_{2} \cap \partial K(\alpha)$. Let us prove the convergence of the integral over $K_{2}$ in (5.8). The other proofs are similar. Indeed,

$$
\begin{aligned}
\infty & >\int_{T}^{\infty} r^{-1-\lambda_{e}} w(r) d r \\
& \geqq \int_{T}^{\infty} \inf _{x \in K(\alpha),|x|=r}\left\{\int_{K(\alpha) n\{|y| \geqq 2 r\}} g(x, y) d \xi(y)\right\} r^{-1-\lambda_{e}} d r \\
& \geqq A_{1} \int_{T}^{\infty}\left\{\int_{K(\alpha) n(|y| \geqq 2 r\}} \psi_{1}(e(y))(r /|y|)^{e}|y|^{2-d} d \xi(y)\right\} r^{-1-\lambda_{e}} d r \\
& \geqq A_{2} \int_{K_{2}} \psi_{1}(e(y))|y|^{2-d-\lambda_{e}} d \xi(y),
\end{aligned}
$$

where $A_{1}$ and $A_{2}$ are positive constants.

In the third inequality we have used (a) of Lemma 2 . In the fourth inequality we have inverted the order of integration which is permissible since all quantities involved are nonnegative.

We now introduce

$$
d \eta(y)= \begin{cases}\psi_{1}(e(y))|y|^{2-d-\lambda_{e}} d \xi(y), & y \in K_{2} \\ \partial \psi_{1}(e(y)) / \partial n|y|^{1-d-\lambda_{e}} d \gamma(y), & y \in \Sigma_{2} \\ \psi_{1}(e(y))|y|^{e} d \xi(y), & y \in K_{1} \\ \partial \psi_{1}(e(y)) / \partial n|y|^{e-1} d \gamma(y), & y \in \Sigma_{1}\end{cases}
$$

and define $N(x, y)$ for $x \in K(\alpha)$ by

$$
N(x, y) d \eta(y)=\left\{\begin{array}{l}
\partial g(x, y) / \partial n d \gamma(y), \quad y \in \partial K(\alpha), \\
g(x, y) d \xi(y), \quad y \in K(\alpha) .
\end{array}\right.
$$


From (5.8) and (5.9) we see that

$$
\int_{\bar{K}(\alpha)} d \eta(y)<\infty
$$

and

$$
p(x)=\int_{K(\alpha)} N(x, y) d \eta(y), \quad x \in K(\alpha) .
$$

Corresponding formulas for the case $\lambda=1$ can be found in Hayman [12, p. 117] and Azarin [2, p. 131].

We now use a method of Hayman and Azarin to prove (A) and (B) of Theorem 1.

\section{Estimates of $N(x, y)$.}

If $x \in K(\alpha)$ is given, let

$$
D(x)=\left\{y \in K(\alpha): \frac{1}{2} \leqq|x| /|y| \leqq 2\right\} .
$$

In the sequel we assume that $|x|>4 T$, so that $D(x) \subset K_{2}$. We also let $A$ denote a positive quantity that may depend only on $\alpha$ and $d$ (note that $\varrho$ is a function of $\alpha$ ), not necessarily the same at each occurrence. If $\alpha_{1}$ is given, $0<\alpha_{1}<\alpha, A\left(\alpha_{1}\right)$ denotes a positive quantity that depends only on $\alpha_{1}, \alpha$, and $d$, not necessarily the same at each occurrence.

If $x \in K(\alpha)$ and $|x|>4 T$, we note that

$$
\begin{gathered}
N(x, y)=g(x, y)\left\{\psi_{1}(e(y))\right\}^{-1}|y|^{d+\lambda_{\ell}-2}, \quad y \in D(x), \\
N(x, y)=\partial g(x, y) / \partial n\left\{\partial \psi_{1}(e(y)) / \partial n\right\}^{-1}|y|^{d+\lambda_{e-1}}, \quad y \in \partial D(x) \cap \partial K(\alpha) .
\end{gathered}
$$

We shall want the following lemma (see Azarin [2, Lemma 4]).

Lemma 3. If $z \in S(\alpha)$, then

$$
g(z, y) \leqq A \psi_{1}(z) \psi_{1}(e(y))|z-y|^{-d}, \quad y \in D(z),
$$

$$
\partial g(z, y) / \partial n \leqq A \psi_{1}(z) \partial \psi_{1}(e(y)) / \partial n|z-y|^{-d}, \quad y \in \partial D(z) \cap \partial K(\alpha) \text {. }
$$

Using (3.2), (3.3), and Lemma 3 we see that

$$
g(x, y) \leqq A \psi_{1}(e(x)) \psi_{1}(e(y))|x|^{2}|x-y|^{-d}
$$

when $x \in K(\alpha), y \in D(x)$, and

$$
\partial g(x, y) / \partial n \leqq A \psi_{1}(e(x)) \partial \psi_{1}(e(y)) / \partial n|x||x-y|^{-d}
$$

when $y \in \partial D(x) \cap \partial K(\alpha)$.

We shall need the following estimates. If $0<\alpha_{1}<\alpha$,

$$
N(x, y) \leqq A\left(\alpha_{1}\right)|x|^{\lambda e}, \quad x \in K\left(\alpha_{1}\right), y \in \partial D(x) \cap \partial K(\alpha) .
$$


Proof. In (6.2) we apply (6.6) and observe that under the assumptions of (6.7), $|x-y| \geqq A\left(\alpha_{1}\right)|x|$.

$$
N(x, y) \leqq A|x|^{d-2}|x-y|^{2-d}\left\{\psi_{1}(e(y))\right\}^{-1}|x|^{\lambda e}
$$

for $x \in K(\alpha)$ and $y \in D(x)$.

Proof. In (6.1) we use the well-known inequality

$$
g(x, y) \leqq|x-y|^{2-d}, \quad x, y \in K(\alpha) .
$$

$$
N(x, y) \leqq A \psi_{1}(e(x))|x|^{d+\lambda \varrho}|x-y|^{-d}, \quad x \in K(\alpha), y \in D(x) .
$$

Proof. In (6.1) we apply (6.5).

\section{The final proof.}

We put

$$
p(x)=\int_{\bar{D}(x)} N(x, y) d \eta(y)+\int_{\bar{R}(x)-\bar{D}(x)} N(x, y) d \eta(y)=I_{1}(x)+I_{2}(x) .
$$

Using Lemma 2 and arguing as in Hayman [12, section 3], it can be shown that $I_{2}(x)=o\left(|x|^{\rho \lambda}\right)$ uniformly in $K(\alpha)$ as $|x| \rightarrow \infty$. Hence it suffices to prove (A) and (B) of Theorem 1 for $I_{1}(x)$. We first prove (A). Let $\alpha_{1}$ be given, $0<\alpha_{1}<\alpha$. Since $\psi_{1}$ is nonnegative it follows from the maximum principle (see [3, p. 326]) that there exists $\omega>0$ such that

$$
\psi_{1}(x)>\omega, \quad x \in S\left(\alpha_{1}\right) .
$$

We claim that for $|x| \geqq 4 T$,

$$
N(x, y) \leqq A\left(\alpha_{1}\right)|e(x)-e(y)|^{2-d}|x|^{\lambda e}, \quad x \in K\left(\alpha_{1}\right), y \in \bar{D}(x) .
$$

To prove (7.2) consider first the case when $\psi_{1}(e(y)) \leqq \frac{1}{2} \omega$. Since $\partial \psi_{1} / \partial \theta_{1}$ is bounded in $S(\alpha)$, we have

$$
\left|\psi_{1}(e(x))-\psi_{1}(e(y))\right| \leqq A|e(x)-e(y)|, \quad x, y \in K(\alpha),
$$

and hence if $\psi_{1}(e(y)) \leqq \frac{1}{2} \omega$,

$$
\omega \leqq A|e(x)-e(y)|, \quad x \in K\left(\alpha_{1}\right) .
$$

From (7.4) and (6.9) it follows that

$$
N(x, y) \leqq A|x|^{\lambda_{\varrho}} \omega^{-2}|e(x)-e(y)|^{2-d}, \quad x \in K\left(\alpha_{1}\right), y \in D(x) .
$$

If $\psi_{1}(e(y)) \geqq \frac{1}{2} \omega$, then by (6.8),

$$
N(x, y) \leqq A \omega^{-1}|e(x)-e(y)|^{2-d}|x|^{\lambda_{e}}, \quad x \in K(\alpha), y \in D(x) .
$$


Since $\omega$ is a function of $\alpha_{1}$, we conclude from (6.7), (7.5), and (7.6) that (7.2) is true.

For given $\varepsilon>0$ and $r>4 T$, let

$$
\begin{aligned}
& \Omega\left(\varepsilon, r, \alpha_{1}\right)=\left\{x: I_{1}(x)>\varepsilon|x|^{\alpha^{\lambda}}\right\} \cap\{|x|>r\} \cap K\left(\alpha_{1}\right), \\
& E\left(\varepsilon, r, \alpha_{1}\right)=\left\{y: y=e(x) \text { for some } x \in \Omega\left(\varepsilon, r, \alpha_{1}\right)\right\} .
\end{aligned}
$$

Let $v$ be a positive Borel measure on $E\left(\varepsilon, r, \alpha_{1}\right)$ of total mass 1. Put

$$
L(v)=\sup _{x \in \mathrm{R}^{d}} \int_{E\left(\varepsilon, r, \alpha_{1}\right)}|x-y|^{2-d} d \nu(y) .
$$

If $x \in \Omega\left(\varepsilon, r, \alpha_{1}\right)$, then from (7.2) we see that

$$
\varepsilon<|x|^{-\varrho \lambda} I_{1}(x) \leqq A\left(\alpha_{1}\right) \int_{K(\alpha) \cap\left\{|y| \geqq \frac{1}{2} r\right\}}|e(x)-e(y)|^{2-d} d \eta(y) .
$$

Integrating this inequality with respect to $v$ and inverting the order of integration, we obtain

$$
\varepsilon<A\left(\alpha_{1}\right) L(v) \int_{K(\alpha) n\left\{|y| \geq \frac{1}{2} r\right\}} d \eta(y) .
$$

Since $\eta\left[K(\alpha) \cap\left\{|y| \geqq \frac{1}{2} r\right\}\right] \rightarrow 0$ as $r \rightarrow \infty$, it follows that

$$
E\left(\varepsilon, \alpha_{1}\right)=\bigcap_{r>4 T} E\left(\varepsilon, r, \alpha_{1}\right)
$$

has capacity zero. Letting $\varepsilon \rightarrow 0$ and $\alpha_{1} \rightarrow \alpha$, we obtain (A) of Theorem 1 for $I_{1}$ and thus for $u$.

We now turn to the proof of (B) of Theorem 1. Following Hayman [12, p. 120] and Azarin [2, p. 133] we make the following definition.

DefinItION. Let $\varepsilon$ be a fixed positive number and suppose that $x \in K(\alpha)$. If for $0<h<\frac{1}{2}|x|$ we have

$$
\int_{K(\alpha) \cap\{|y-x|<h\}} d \eta(y)<\varepsilon(h /|x|)^{d-1},
$$

then $x$ is said to be $\varepsilon$ normal with respect to $\eta$.

Also using the technique of Azarin [2, Lemma 6] we prove

Lemma 4. If $x$ is $\varepsilon$ normal and $|x|>4 T$, then

$$
I_{1}(x) \leqq(A \varepsilon+o(1))|x|^{\rho \lambda}, \quad|x| \rightarrow \infty .
$$

Proof. Let $x$ be $\varepsilon$ normal and suppose that $|x|>4 T$. We put

where

$$
J_{1}(x)=\int_{D_{1}(x)} N(x, y) d \eta(y),
$$

$$
D_{1}(x)=K(\alpha) \cap\left\{y:|y-x| \leqq \frac{1}{2}|x|\right\} .
$$


If $I_{1}(x)=J_{1}(x)+J_{2}(x)$, it follows from (6.1), (6.2), (6.5), and (6.6) that

$$
J_{2}(x) \leqq A\left\{\int_{\bar{D}(x)} d \eta(y)\right\}|x|^{\rho^{\lambda}}, \quad x \in K(\alpha),|x|>4 T .
$$

To estimate $J_{1}$, we define, if $n$ is an integer

$$
\begin{aligned}
B_{n}=\{y: & \left.|y-x| \leqq \frac{1}{2}|x|\right\} \cap \\
& \cap\left\{y \in \bar{K}(\alpha): 2^{n-1}|x| \psi_{1}(e(x)) \leqq|x-y|<2^{n}|x| \psi_{1}(e(x))\right\}
\end{aligned}
$$

Since $x$ is $\varepsilon$ normal, $\eta(\{x\})=0$ and

$$
J_{1}(x)=\sum_{-\infty}^{\infty} \int_{B_{n}} N(x, y) d \eta(y), \quad x \in K(\alpha),|x|>4 T .
$$

Let $C$ denote the constant in (7.3). Let $n_{0}$ be the least positive integer such that $C 2^{-n_{0}+1}<1$. We note that

$$
|e(x)-e(y)| \leqq 2|x|^{-1}|x-y|, \quad x, y \neq 0 .
$$

If $y \in \cup_{n \leqq-n_{0}} B_{n}$, it follows from (7.9) and (7.3) that

$$
\psi_{1}(e(x))-\psi_{1}(e(y)) \leqq 2 C|x|^{-1}|x-y| \leqq 2^{-n_{0}+1} C \psi_{1}(e(x)),
$$

and so

$$
\psi_{1}(e(y)) \geqq A \psi_{1}(e(x)), \quad y \in \bigcup_{n \leqq-n_{0}} B_{n} .
$$

Using (7.10) we estimate $N(x, y)$ when $y \in B_{n}, n \leqq-n_{0}$. Indeed in this case we see from (6.8) and (7.10) that

$$
N(x, y) \leqq A 2^{(n-1)(2-d)} \psi_{1}(e(x))^{1-d}|x|^{e^{\lambda}} .
$$

Since $x$ is $\varepsilon$ normal, it follows that if $h=\min \left\{\frac{1}{2}|x|, 2^{n} \psi_{1}(e(x))|x|\right\}$,

$$
\int_{B_{n}} N(x, y) d \eta(y) \leqq A \varepsilon 2^{n}|x|^{e^{\lambda}}, \quad n \leqq-n_{0} .
$$

If $n>-n_{0}$, we first suppose that $y \in K(\alpha) \cap B_{n}$. Then from (6.9) we find that

$$
N(x, y) \leqq A \psi_{1}(e(x))^{1-d} 2^{(1-n) d}|x|^{e^{\lambda}}, \quad x \in K(\alpha) .
$$

If $y \in \partial K(\alpha) \cap B_{n}$, then (7.12) is also true, as follows from (6.6).

Using (7.12) and the fact that $x$ is $\varepsilon$ normal, we obtain

$$
\int_{B_{n}} N(x, y) d \eta(y) \leqq A \varepsilon 2^{-n}|x|^{e^{\lambda}}, \quad n>-n_{0} .
$$

Summing over $n$ in (7.11) and (7.13), we deduce that Lemma 4 is true for $J_{1}$. Since $\eta[\bar{D}(x)] \rightarrow 0$ as $|x| \rightarrow \infty$, it follows from (7.7) that Lemma 4 also holds for $I_{1}$.

To conclude the proof of (B) of Theorem 1 for $I_{1}$, we shall want the following lemma of Azarin [2, Lemma 7]. 
LeMma 5. The set $\Delta(\varepsilon)$ of points not $\varepsilon$ normal may be covered by a system $F(\varepsilon)$ of spheres $\left\{G_{i}\right\}$ whose radii $\left\{r_{i}\right\}$ and distances $\left\{R_{i}\right\}$ from their centers to the origin satisfy

$$
\sum_{i=1}^{\infty}\left(r_{i} / R_{i}\right)^{d-1}<\infty
$$

REMARK. Azarin proves Lemma 5 by using a lemma of Landkof. A proof of Landkof's result can be found in [15, Lemma 3.2].

The rest of the proof is similar to Azarin's proof. From Lemma 5 we see there exists a sequence of increasing positive numbers $t_{n} \rightarrow \infty, n \geqq 1$, such that for $G_{i} \in F\left(n^{-1}\right)$ and $R_{i}>t_{n}$,

$$
\sum_{R_{i}>t_{n}}\left(r_{i} / R_{i}\right)^{d-1}<2^{-n} .
$$

Let $F\left(n^{-1}, t_{n}\right)$ denote the set of spheres whose radii appear in the above sum and put

$$
F_{0}=\bigcup_{n=1}^{\infty} F\left(n^{-1}, t_{n}\right) \text {. }
$$

Then clearly for the spheres in $F_{0}$ we have $\Sigma_{i}\left(r_{i} / R_{i}\right)^{d-1}<1$. Moreover if $x \in K(\alpha),|x| \geqq t_{n}$, and $x$ does not belong to one of the spheres in $F_{0}$, then from Lemma 4 we have

$$
I_{1}(x) \leqq\left[A n^{-1}+o(1)\right]|x|^{\lambda_{e}} .
$$

We conclude from the above inequality that (B) of Theorem 1 is valid for $I_{1}$. From our previous reductions it now follows that (B) holds for $u$. This completes the proof of Theorem 2.

\section{On estimates of eigenfunctions.}

In the proof of Lemma 2, we used the following estimate of a normalized eigenfunction of (1.2). If $d \geqq 6$,

$$
|\varphi(x)| \leqq A \mu^{(d+1) / 4} \operatorname{dist}(x, \partial S(\alpha)),
$$

where $\mu$ is the corresponding eigenvalue. The constant $A$ depends only on the domain $S(\alpha)$ and $d$. If $2 \leqq d \leqq 5$, similar estimates are valid. They are deduced using the same method of proof as in the case $d \geqq 6$.

We start from the following estimates of the Green's function, $g$, for a second-order uniformly elliptic operator with Hölder continuous coefficients in a bounded $C^{2}$ domain $\Omega$ in $\mathrm{R}^{q}(\operatorname{In}(8.1), q=d-1)$. They are implicit in Widman [18a]. An explicit proof is given in Widman [18b]. The constant $A$ in (8.2) and (8.3) and in the sequel depends only on the ellipticity constants, the Hölder constants, $\Omega$, and $q$. It is not necessarily the same at each occurrence. 


$$
\begin{gathered}
g(x, y) \leqq A|x-y|^{2-q}, \quad x, y \in \Omega, \\
g(x, y) \leqq A d(x) d(y)|x-y|^{-q}, \quad x, y \in \Omega,
\end{gathered}
$$

where $d(x)=\operatorname{dist}(x, \partial \Omega)$.

We want an estimate of $M=\sup _{y \in \Omega}|\varphi(y)| / d(y)$. For that purpose we choose a point $x$ such that $|\varphi(x)| / d(x) \geqq \frac{1}{2} M$.

Let $\delta>0$ be given and suppose that $d(x)<\delta$. The case $d(x) \geqq \delta$ will be discussed later. We define

Since

$$
\begin{aligned}
& D_{1}=\{y:|y-x|<d(x)\} \cap \Omega, \\
& D_{2}=\{y: d(x) \leqq|y-x|<\delta\} \cap \Omega, \\
& D_{3}=\{y:|y-x| \geqq \delta\} \cap \Omega .
\end{aligned}
$$

it is clear that

$$
\varphi(x)=\mu \int_{\Omega} g(x, y) \varphi(y) d y
$$

$$
\frac{\varphi(x)}{d(x)}=\mu \int_{\Omega} \frac{\varphi(y)}{d(y)} \frac{d(y)}{d(x)} g(x, y) d y .
$$

We claim that if $q \geqq 3$,

$$
\begin{aligned}
\left|\int_{D_{1}} d(y) g(x, y) d y\right| & \leqq A d(x)^{3} \\
\left|\int_{D_{2}} d(y) g(x, y) d y\right| & \leqq A d(x) \delta^{2} \\
\left|\int_{D_{3}} \varphi(y) g(x, y) d y\right| & \leqq A d(x) \delta^{1-q / 2}
\end{aligned}
$$

Proof of (8.5). Applying (8.2), we see that

$$
\begin{gathered}
\left|\int_{D_{1}} d(y) g(x, y) d y\right| \leqq A \int_{D_{1}}(d(x)+|x-y|)|x-y|^{2-q} d y \\
\leqq A \int_{0}^{d(x)} d(x) \varrho d \varrho \leqq A d(x)^{3} .
\end{gathered}
$$

Proof of (8.6). Applying (8.3), we see that

$$
\begin{gathered}
\left|\int_{D_{\mathbf{2}}} d(y) g(x, y) d y\right| \leqq A \int_{D_{\mathbf{2}}} d(y)^{2} d(x)|x-y|^{-q} d y \\
\leqq A d(x) \int_{d(x)}^{\delta} \varrho \varrho \varrho \leqq A d(x) \delta^{2} .
\end{gathered}
$$

Proof of (8.7). Since $\int_{\Omega}|\varphi(y)|^{2} d y=1$, it follows from (8.3) that

$$
\begin{aligned}
\left\{\int_{D_{3}} \varphi(y) g(x, y) d y\right\}^{2} & \leqq \int_{D_{3}} g(x, y)^{2} d y \\
& \leqq A \int_{D_{3}} d(x)^{2} d(y)^{2}|x-y|^{-2 q} d y \\
& \leqq A d(x)^{2} \int_{\delta}^{A} \varrho^{1-q} d \varrho \leqq A d(x)^{2} \delta^{2-q} .
\end{aligned}
$$

From our choice of $x$ and (8.4)-(8.7), we see that

$$
M \leqq A\left\{\mu M\left(d(x)^{2}+\delta^{2}\right)+\delta^{1-q / 2} \mu\right\},
$$


which is equivalent to

$$
M \leqq A \mu \delta^{1-\alpha / 2}\left\{1-A \mu\left(d(x)^{2}+\delta^{2}\right)\right\}^{-1} .
$$

If $d(x) \leqq \frac{1}{2}(A \mu)^{-\frac{1}{2}}$, we choose $\delta=\frac{1}{2}(A \mu)^{-\frac{1}{3}}$ and it follows that

$$
M \leqq A \mu^{\frac{1}{2}+q / 4},
$$

and thus (8.1) is proved in this case.

If

$$
d(x) \geqq \frac{1}{2}(A \mu)^{-\frac{1}{2}}=\delta,
$$

we argue in the following way. Define, if $\eta>0$ is given,

$$
\begin{aligned}
& D_{1}=\{y:|y-x|<\eta\} \cap \Omega, \\
& D_{2}=\{y:|y-x| \geqq \eta\} \cap \Omega .
\end{aligned}
$$

We claim that if $q \geqq 3$,

$$
\left|\int_{D_{1}} d(y) g(x, y) d y\right| \leqq A \eta^{2}(\eta+d(x)),
$$

and that if $q \geqq 5$,

$$
\left|\int_{D_{2}} \varphi(y) g(x, y) d y\right| \leqq A \eta^{2-q / 2} .
$$

Proof of (8.9). Apply (8.2) in the same way as in the proof of (8.5).

Proof of (8.10). Apply (8.2) and argue in the same way as in the proof of (8.7).

It follows from our choice of $x,(8.4)$, and (8.8)-(8.10) that

$$
\begin{aligned}
M & \leqq A\left\{M \mu\left(\eta^{3}(d(x))^{-1}+\eta^{2}\right)+\mu \eta^{2-q / 2}(d(x))^{-1}\right\} \\
& \leqq A\left\{M\left(\mu^{3 / 2} \eta^{3}+\mu \eta^{2}\right)+\mu^{3 / 2} \eta^{2-q / 2}\right\},
\end{aligned}
$$

which is equivalent to

$$
M \leqq A \mu^{3 / 2} \eta^{2-q / 2}\left\{1-A \eta^{2}\left(\mu+\eta \mu^{3 / 2}\right)\right\}^{-1} .
$$

Choosing $\eta=\frac{1}{2}(A \mu)^{-1}$, we see that

$$
M \leqq A \mu^{1+q / 4},
$$

and thus (8.1) is true also in this case. This completes the proof of (8.1). 


\section{REFERENCES}

1. L. Ahlfors and M. Heins, Questions of regularity connected with the Phragmen-Lindelof principle, Ann. of Math. 50 (1949), 341-346.

2. V. Azarin, Generalization of theorem of a Hayman on subharmonic functions in an n-di. mensional cone, Amer. Math. Soc. Transl. (2) 80 (1969), 119-138. Mat. Sb. 66 (108), (1965), 248-264.

3. R. Courant and D. Hilbert, Methods of mathematical physics, II, Interscience Publishers Inc., New York, London, 1962.

4. B. Dahlberg, Growth properties of subharmonic functions, thesis, University of Göteborg, 1971.

5. M. Essén, Note on " $A$ theorem on the minimum modulus of entire functions" by Kjellberg, Math. Scand. 12 (1963), 12-14.

6a. M. Essén, A generalization of the Ahlfors-Heins theorem, Bull. Amer. Math. Soc. 75 (1969), 127-131.

6b. M. Essén, A generalization of the Ahlfors-Heins theorem, Trans. Amer. Math. Soc. 142 (1969), 331-344.

7. M. Essén and J. L. Lewis, The generalized Ahlfors-Heins theorem in certain d-dimensional cones, Conference on the theory of ordinary and partial differential equations, Dundee, March 1972, (Lecture Notes in Mathematics 280), 246-257, SpringerVerlag, Berlin, Heidelberg, New York, 1972.

8. M. Essén, The generalized Ahlfors-Heins theorem in $\mathbf{R}^{3}$, to appear in Proceedings of a conference on mathematical analysis at Jyväskylä, Finland, August 1970, (Lecture Notes in Mathematics), Springer-Verlag, Berlin, Heidelberg, New York.

9. J. Lelong-Ferrand, Étude au voisinage de la frontière des fonctions subharmoniques positives dans un demi-espace, Ann. Sci. École Norm. Sup. (3) 66 (1949), 125-159.

10. J. Lelong-Ferrand, Étude des fonctions surharmoniques positives dans un cylindre ou dans un cóne, C. R. Acad. Sci Paris Sér A 229 (1949), 340-341.

11. J. Lelong-Ferrand, Extension du théorème de Phragmén-Lindelöf-Heins aux fonctions sousharmoniques dans un cóne ou dans un cylindre, C. R. Acad. Sci. Paris Sér. A 229 (1949), 411-413.

12. W. Hayman, Questions of regularity connected with the Phragmen-Lindelof principle, J. Math. Pure Appl. (9) 35 (1956), 115-126.

13. M. Heins, Selected topics in the classical theory of functions of a complex variable, Holt, Rinehart and Winston, New York, 1962.

14. L. L. Helms, Introduction to potential theory (Pure and Applied Mathematics 22), Wiley-Interscience, New York, London, 1969.

15. N. S. Landkof, Foundations of modern potential theory, (Russian) Izdatel'stra "Nauka" Moscow 1966. (Grundlehren Math. Wiss. 180) Springer-Verlag, Berlin, New York, 1972.

16. J. L. Lewis, Subharmonic functions in certain regions, Trans. Amer. Math. Soc. 167 (1972), 191-201.

17. J. L. Lewis, On Essén's generalization of the Ahlfors-Heins theorem, Trans. Amer. Math. Soc. 172 (1972), 339-345.

18a. K. O. Widman, Inequalities for the Green function and boundary continuity of the gradient of solutions of elliptic differential equations, Math. Scand. 21 (1967), 17-37.

18b. K. O. Widman, Inequalities for Green functions of second order elliptic operators, Report nr. 8, 1972, Department of Mathematics, Linköping University, S-58183 Linköping, Sweden.

ROYAL INSTITUTE OF TECHNOLOGY, STOCKHOLM, SWEDEN

AND

UNIVERSITY OF KENTUCKY, LEXINGTON, KENTUCKY, U.S.A.

Math. Scand. 33 - 9 